\title{
Covariant perturbations in the gonihedric string model
}

\author{
Efraín Rojas* \\ Facultad de Física, Universidad Veracruzana, Cto. Gonzálo Aguirre Beltrán s/n, Xalapa, Veracruz 91000, México
}

\begin{abstract}
We provide a covariant framework to study classically the stability of small perturbations on the so-called gonihedric string model by making precise use of variational techniques. The local action depends of the square root of the quadratic mean extrinsic curvature of the worldsheet swept out by the string, and is reparametrization invariant. A general expression for the worldsheet perturbations, guided by Jacobi equations without any early gauge fixing, is obtained. This is manifested through a set of highly coupled nonlinear differential partial equations where the perturbations are described by scalar fields, $\Phi^{i}$, living in the worldsheet. This model contains, as a special limit, to the linear model in the mean extrinsic curvature. In such a case the Jacobi equations specialize to a single wave-like equation for $\Phi$.
\end{abstract}

PACS numbers: $04.20 . \mathrm{Fy}, 11.25 .-\mathrm{w}, 46.70 . \mathrm{Hg}$

\section{INTRODUCTION}

The so-called gonihedric string model is considered as a natural extension of the Feynman path integral over random walks, to an integral over random surfaces. The action functional is defined in a way that, when the worldsdsheet swept out by a closed string, degenerates into a worldline such an action must be reduced to an action of a point-like relativistic particle [1-3]. This field theory, at classical level, predicts the existence of tensionless strings possessing a massless spectrum of higher integer spin gauge fields [4 6] whereas, at quantum level, fluctuations generate a nonzero string tension [1 3]. Moreover, when the theory is formulated on an Euclidean lattice it has a close relationship with a spin system which generalizes the Ising model with ferromagnetic, antiferromagnetic and quartic interactions [7-9].

The effective action for the theory is proportional to the linear size of a random surface. Under this assumption, in building the model, a dimensional analysis as a guide unavoidably entails that the concept of extrinsic curvature associated to the surface must be included [13]. More specifically, on geometrical grounds, the model looks like an extrinsic volume through of the modulus of the mean extrinsic curvature, $K^{i}$, where $i$ stands for the number of normal vectors of the worldsheet immersed into a background spacetime. While the associated equations of motion (eom) resemble wave-like equations for a unit vector, $\widehat{K}^{i}$, in the normal frame of the theory, this apparent advantage is deceptive since the $\widehat{K}^{i}$ are derived objects constructed from the physical field variables, that is, the embedding functions $X^{\mu}$. In terms of these, the eom are of fourth-order, which are intractable unless a high degree of symmetry is consider on the surface be considered. Even if mathematically a solution exists for this type of surfaces, the possibility of it being a physical object in nature depends on whether it is stable or not. Regarding this point, an elegant description for examin-

\footnotetext{
* efrojas@uv.mx
}

ing the stability of a geometric object is provided by a manifestly covariant analysis.

This paper, motivated mainly from some recent reviews [9, 10], is devoted specifically to obtain a manifestly covariant expression to describe the classically perturbations in a relativistic surface described by the gonihedric string model. Unlike some perturbative analyzes [4, 5, 11 ] for this model, our emphasis is on the worldsheet geometry by taking advantage of the inherent geometric structures defined on the worldsheet. The perturbation analysis we develop is about classical solutions with the advantage of bringing to the foreground how the covariance under rotations of the normals to the worldsheet enters into the game. In my opinion, this issue was overlooked in several contributions and does not appear to have been addressed before. Additionally, our analysis is performed without impose any early gauge fixing.

Following the guideline for brane theories deformations, it is known that for an extended object of arbitrary dimension, $p$, in building local actions using invariants characterizing the geometry of the associated $(p+1)$-dimensional worldvolume, at least three ingredients are mandatory: invariance under reparametrizations of the worldvolume, diffeomorphisms invariance of the background spacetime, and, when $p+1<N$, invariance under rotations of the normal vectors adapted to the worldvolume [12, 13]. This last fact is necessary to identify that in the geometric extrinsic structure description, we have the presence of a connection that guarantee the covariance under worldvolume normal rotations. Needless to say, we must take into account these facts if we try to understand deeply the geometrical underpinnings of the gonihedric relativistic model. A classically equivalent action, close in spirit to the one developed by $\mathrm{G}$. Savvidy and collaborators, to describe tensionless strings was performed in 14, 15].

Our aim is twofold. First, we highligth the geometric content of the model. In particular, we emphasize the covariance of the description not only with respect to worldsheet diffeomorphisms but also with respect to local rotations of the normals to the worldsheet since we have a codimension of the worldsheet greater than one. 
Second, we obtain a consistent covariant derivation of the linearized equations of motion about classical solutions and reach the Jacobi equations describing the perturbations of the worldsheet governed by the gonihedric string action. These are highly coupled partial differential equations for a multiplet of scalars fields, $\Phi^{i}$, with support on the worldsheet.

The paper is organized as follows. In Sect. [II we briefly review the gonihedric string model and emphasize the role that the extrinsic geometry plays in its description. After established the notation and conventions, in Sect. III we perform the first variation of the action and identify the equations of motion. In Sect. [V] we achieve the second variation of the action and thereby we obtain the linearized equations of motion which are nothing but the conditions for stability of this type of surfaces. We conclude in Sec. $\mathrm{V}$ with some comments of the work.

\section{THE GONIHEDRIC STRING MODEL}

The gonihedric string action is defined by the functional [1-6]

$$
S\left[X^{\mu}\right]=\alpha \int_{m} d^{2} x \sqrt{-g} \sqrt{K^{i} K_{i}},
$$

where $X^{\mu}$ represents the field variables which correspond to the embedding functions of the smooth worldsheet, denoted by $m$, swept out by a closed string when evolving in a Minkowski $N$-dimensional spacetime, denoted by $\mathcal{M}$, with metric $\eta_{\mu \nu}=\operatorname{diag}(-1,1.1, \ldots, 1)$ where $\mu, \nu=0,1,2, \ldots, N-1$. To specify the string trajectory we set $x^{\mu}=X^{\mu}\left(x^{a}\right)$, where $x^{\mu}$ are local coordinates in the spacetime, $x^{a}$ are coordinates on the worldsheet $(a, b=0,1)$, and $\alpha$ is a constant with appropriate dimensions. Further, $g=\operatorname{det}\left(g_{a b}\right)$ and $K^{i}$ stands for the determinant of the induced metric on $m$ and the trace of the $i$-th extrinsic curvature $(i, j=1,2, \ldots, N-2)$, respectively, (see below for details).

The two vectors $e^{\mu}{ }_{a}:=\partial_{a} X^{\mu}$ form a basis of tangent vectors to $m$. These allow to define an induced metric on $m$ as $g_{a b}:=e_{a} \cdot e_{b}$. Hereafter, a central dot will denote contraction with the Minkowski metric. In addition, $\nabla_{a}$ will denote the (torsionless) covariant derivative compatible with $g_{a b}$. Similarly, the $i$-th normal vector to $m, n^{\mu i}$, is defined by the relations

$$
e_{a} \cdot n^{i}=0, \quad n^{i} \cdot n^{j}=\delta^{i j},
$$

where $\delta^{i j}$ is the Kronecker delta. It is worth to note that these expressions determine $n^{\mu i}$ only up to a $O(N-2)$ rotation (and a sign) and it transforms as a vector under normal frame rotations [16, 17]. Whereas the tangential indices are lowered and raised with $g_{a b}$ and $g^{a b}$, respectively, the normal indices are lowered and raised with $\delta_{i j}$ and $\delta^{i j}$, respectively.

The gradients of the orthonormal basis entail the definition of the extrinsic curvature tensor, $K_{a b}^{i}=-n^{i}$. $\nabla_{a} \nabla_{b} X=K_{b a}^{i}$. Apart from the extrinsic curvature, the extrinsic geometry of a surface, when the codimension is higher than one, is complemented with the extrinsic twist potential, $\omega_{a}^{i j}$, defined by

$$
\omega_{a}^{i j}:=\nabla_{a} n^{i} \cdot n^{j}=-\omega_{a}^{j i}
$$

In this sense, the so-called Gauss-Weingarten equations describing an embedded timelike worldsheet in a spacetime are given by $\nabla_{a} e^{\mu}{ }_{b}=-K_{a b}^{i} n^{\mu}{ }_{i}$ and $\nabla_{a} n^{\mu i}=$ $K_{a}{ }^{b i} e^{\mu}{ }_{b}+\omega_{a}^{i j} n^{\mu}{ }_{j}$. In the case of a hypersurface embedding, $i=1$, the extrinsic twist vanishes identically. Under a rotation $n^{i} \rightarrow O^{i}{ }_{j} n^{j}$, this potential transforms as a connection so that, this quantity is considered as the gauge field associated with the normal frame rotation group [18]. Therefore, to implement normal frame covariance in a manifest way, we need the existence of a new covariant derivative $\widetilde{\nabla}_{a}$, defined on fields transforming as tensors under normal frame rotations [16 18], $\widetilde{\nabla}_{a} \Phi^{i}{ }_{j}:=\nabla_{a} \Phi^{i}{ }_{j}-\omega_{a}^{i k} \Phi_{k j}-\omega_{a j k} \Phi^{i k}$. This fact signals the presence of a curvature associated with the twist defined by $\Omega_{a b}{ }^{i j}:=\nabla_{b} \omega_{a}^{i j}-\nabla_{a} \omega_{b}^{i j}-\omega_{b}^{i k} \omega_{a k}{ }^{j}+\omega_{a}^{i k} \omega_{b k}{ }^{j}$.

Some remarks are in order. First, it must be stressed that the action (11) is proportional to the lenght of the surface. Geometrically, this corresponds to the linear size of the surface, as it was for the path integral [1 3]. Second, in Ref. 19] Savvidy considered the gonihedric model in two classically equivalent theories: The model $A$, when the independent field variables are the embedding functions $X^{\mu}$, and the model $B$ in which both $X^{\mu}$ and the induced metric $g_{a b}$ are the independent variables. In this parlance, in our geometrical approach we will develop further the so-called model $A$.

\section{FIRST VARIATION AND THE EQUATIONS OF MOTION}

In order to pave the way to obtain the stability conditions for this type of surfaces we opt to directly vary the main geometric quantities involved. The variation of the action (11) is

$$
\delta S=\alpha \int_{m} d^{2} x\left(\delta \sqrt{-g} \sqrt{K^{i} K_{i}}+\sqrt{-g} \delta \sqrt{K^{i} K_{i}}\right) .
$$

Carrying out the variations under the integral sign we have first that $\delta \sqrt{-g}=(\sqrt{-g} / 2) g^{a b} \delta g_{a b}$. Regarding the second term we have $\delta \sqrt{K^{i} K_{i}}=\frac{K^{i}}{\sqrt{K^{j} K_{j}}} \widetilde{\delta} K_{i}$. Here, we have considered the deformation operator, $\widetilde{\delta}$, covariant under normal frame rotations, constructed in analogy to the covariant derivative $\widetilde{\nabla}_{a}[20]$. We requiere also that $\widetilde{\delta} K^{i}=\widetilde{\delta}\left(g^{a b} K_{a b}^{i}\right)=\delta g^{a b} K_{a b}^{i}+g^{a b} \widetilde{\delta} K_{a b}^{i}=$ $-K^{a b i} \delta g_{a b}+g^{a b} \widetilde{\delta} K_{a b}^{i}$. These relations allow us to write 
the variation (4) in the form

$$
\begin{aligned}
\delta S & =\alpha \int_{m} d A\left\{\widehat { K } _ { i } \left[\left(\frac{1}{2} g^{a b} K^{i}-K^{a b i}\right) \delta g_{a b}\right.\right. \\
& \left.\left.+g^{a b \widetilde{\delta}} K_{a b}^{i}\right]\right\},
\end{aligned}
$$

where the dependence of the variations of the first and second fundamental forms, $g_{a b}$ and $K_{a b}^{i}$, respectively, is explicitly manifested, and where we have also introduced the unit vector, $\widehat{K}^{i}:=\frac{K^{i}}{\sqrt{K^{j} K_{j}}}$. This vector may be thought of as being the coordinates of a $S^{(N-2)}$ unit sphere, $\delta_{i j} \widehat{K}^{i} \widehat{K}^{j}=1$. Evidently, $\widehat{K}^{i}$ transforms like a vector under normal frame rotations. Additionally, hereafter, we will use $d A:=d^{2} x \sqrt{-g}$, for short in the notation.

The infinitesimal changes of the field variables, $X^{\mu}\left(x^{a}\right) \rightarrow X^{\mu}\left(x^{a}\right)+\delta X^{\mu}\left(x^{a}\right)$, can be decomposed into tangential and normal deformations, that is, $\delta X^{\mu}=$ $\Phi^{a} e^{\mu}{ }_{a}+\Phi^{i} n^{\mu}{ }_{i}$ where $\Phi^{a}$ and $\Phi^{i}$ denote both tangential and normal deformation fields, respectively. On the other hand, the fact that the gauge symmetry of the action (11) is the invariance under reparametrizations of the worldsheet, it determines that only the transverse worldsheet motion is physical so that the tangential deformations, $\delta_{\|} X^{\mu}=\Phi^{a} \partial_{a} X^{\mu}$, are usually ignored [20]. Therefore, we will only consider $\delta_{\perp} X^{\mu}=\Phi^{i} n^{\mu}{ }_{i}$, where the $\Phi^{i}$ are assumed to be functions of $x^{a}$. Consequently, according to the geometric approach introduced in Ref. [20], the variations of the fundamental forms are given by the simple expressions

$$
\begin{aligned}
\delta_{\perp} g_{a b} & =2 K_{a b}^{i} \Phi_{i}, \\
\widetilde{\delta}_{\perp} K_{a b}^{i} & =-\widetilde{\nabla}_{a} \widetilde{\nabla}_{b} \Phi^{i}+K_{a c}^{i} K_{b j}^{c} \Phi^{j} .
\end{aligned}
$$

In terms of these, Eq. (5) becomes

$$
\delta_{\perp} S=-\alpha \int_{m} d A\left[\widetilde{\Delta} \widehat{K}^{i}-\left(g^{a b} K_{j}-K_{j}^{a b}\right) K_{a b}^{i} \widehat{K}^{j}\right] \Phi_{i},
$$

up to a total derivative where $\widetilde{\Delta}=g^{a b} \widetilde{\nabla}_{a} \widetilde{\nabla}_{b}$ denotes the worldsheet d'Alembertian operator. Hence, the classical string trajectories are obtained from the $N-2$ relations

$$
\mathcal{E}^{i}=\widetilde{\Delta} \widehat{K}^{i}-J_{j}^{a b} K_{a b}^{i} \widehat{K}^{j}=0,
$$

where we have introduced the symmetric tensor

$$
J_{i}^{a b}:=g^{a b} K_{i}-K_{i}^{a b} .
$$

This is conserved in the sense that $\widetilde{\nabla}_{a} J_{i}^{a b}=0$ which is courtesy of the Codazzi-Mainardi integrability condition for surfaces when the background spacetime is Minkowski [21]. For future convenience, we also introduce the symmetric tensor in the normal frame

$$
R_{i j}:=J_{i}^{a b} K_{a b j}=R_{j i} .
$$

Clearly, $\operatorname{Tr}\left(R_{i j}\right)=\delta^{i j} R_{i j}=K^{i} K_{i}-K_{a b}^{i} K_{i}^{a b}=\mathcal{R}$, the worldsheet Ricci scalar, which is nothing but the contracted Gauss-Codazzi integrability condition in a flat spacetime background. In terms of the tensor (11), the eom (9) can be written in the fashion

$$
\mathcal{E}^{i}=\widetilde{\Delta} \widehat{K}^{i}-R_{j}^{i} \widehat{K}^{j} .
$$

On pedagogical grounds, this set of equations can be seen as a set of wave-like equations for the variables $\widehat{K}^{i}$ but we must have in mind that the field variables are the embedding functions. Regarding this point, regrettably, the eom (9) are of fourth-order in the derivatives of $X^{\mu}$.

Hence, at this stage we have that under the deformation $X^{\mu} \rightarrow X^{\mu}+\delta X^{\mu}$, the first variation of the action (1) reads

$$
\delta_{\perp} S=-\alpha \int_{m} d A\left(\widetilde{\Delta} \widehat{K}_{i}-R_{i j} \widehat{K}^{j}\right) \Phi^{i},
$$

where (12) is the result of the physical transverse motion provided by the breathing modes, $\Phi^{i}$, living in the worldsheet $m$.

Concerning the hypersurface embedding case, $i=1$, the action (11) specializes to a functional depending linearly of the mean extrinsic curvature which has been discussed extensively in the relativistic context in the framework of the Lovelock type branes [22, 23] whereas in the Euclidean context such functional has attracted lot of attention as being part of the geometrical prescription to study biological lipid membranes 25-28]. In such a case, $R_{i j}$ specializes to $\mathcal{R}$ so that, $\widehat{K}^{(1)}=1$ and the equations of motion (12) reduce to a single equation of second order in the derivatives of the fields, $\mathcal{E}_{(1)}=\mathcal{R}=0$. In other words, we recuperate the case of the action extremized by worldsheets with vanishing Ricci scalar curvature.

\section{SECOND VARIATION AND THE LINEARIZED EQUATIONS OF MOTION}

The way we follow to obtain the linearized equations of motion is to exploit the relations given by (6) and (7). As discussed formally in Ref. [25], the computation of the second variation of an action for surfaces simplifies enormously when the Euler-Lagrange equations are satisfied. There, has been proved that the calculation of the second variation is equivalent to a repeated application of the normal deformation operator, $\widetilde{\delta}_{\perp}$. A related approach about the analysis of the stability for minimal surfaces was developed long time ago by H. A. Schwarz in [30]. In this sense, from (13), let us consider the second variation of the action (1)

$$
\delta^{2} S_{\perp}=\int_{m} d^{2} x \delta_{\perp}\left(\sqrt{-g} \mathcal{E}_{i} \Phi^{i}\right),
$$

where, modulo the eom, it follows that the relevant equation to be exercised is

$$
\widetilde{\delta}_{\perp} \mathcal{E}_{i}=\widetilde{\delta}_{\perp}\left(\widetilde{\Delta} \widehat{K}_{i}-R_{i j} \widehat{K}^{j}\right) .
$$


Clearly, we note that three specific variations are involved, $\widetilde{\delta}_{\perp} \widehat{K}^{i}, \widetilde{\delta}_{\perp} R_{i j}$ and $\widetilde{\delta}_{\perp}\left(\widetilde{\Delta} \widehat{K}^{i}\right)$ so that we perform these in steps. Guided by (마) and (7) we get

$$
\begin{aligned}
\delta_{\perp} g^{a b} & =-2 K_{i}^{a b} \Phi^{i}, \\
\widetilde{\delta}_{\perp} K_{i}^{a b} & =-\widetilde{\nabla}^{a} \widetilde{\nabla}^{b} \Phi_{i}-3 K^{(a}{ }_{c i} K_{j}^{b) c} \Phi^{j}, \\
\widetilde{\delta}_{\perp} K^{i} & =-\widetilde{\Delta} \Phi^{i}+\left(R_{j}^{i}-K^{i} K_{j}\right) \Phi^{j},
\end{aligned}
$$

where we have considered the fact that $K_{a b i} K_{j}^{a b}=$ $-R_{i j}+K_{i} K_{j}$. Hence, it is straightforward to compute the variation of the unit vector $\widehat{K}^{i}$

$$
\widetilde{\delta}_{\perp} \widehat{K}^{i}=-\frac{1}{\sqrt{K^{r} K_{r}}} \Pi^{i j}\left(\widetilde{\Delta} \Phi_{j}-R_{j l} \Phi^{l}\right),
$$

where we have introduced the projection operator

$$
\Pi^{i j}:=\delta^{i j}-\widehat{K}^{i} \widehat{K}^{j},
$$

satisfying

$$
\Pi^{i}{ }_{j} \widehat{K}^{j}=0 \quad \text { and } \quad \Pi^{i}{ }_{j} \widetilde{\nabla}_{a} \widehat{K}^{j}=\widetilde{\nabla}_{a} \widehat{K}^{i} .
$$

These results together with the unit vector fact $\widehat{K}_{i} \widetilde{\nabla}_{a} \widehat{K}^{i}=0$, provide that $\left\{\widetilde{\nabla}_{a} K^{i}, \widehat{K}^{i}\right\}$ is an orthonormal basis for a unit sphere $S^{(N-2)}$. Notice that for the case of a hypersurface, $i=1$, we have that $\Pi^{i j}$ vanishes. Similarly, a forthright computation leads to

$$
\begin{aligned}
\widetilde{\delta}_{\perp} J_{i}^{a b} & =-g^{a b} \widetilde{\Delta} \Phi_{i}+\widetilde{\nabla}^{a} \widetilde{\nabla}^{b} \Phi_{i}+3 K^{(a}{ }_{c i} K_{j}^{b) c} \Phi^{j} \\
& +2 K_{i} J_{j}^{a b} \Phi^{j}+g^{a b} R_{i j} \Phi^{j}-3 g^{a b} K_{i} K_{j} \Phi^{j}
\end{aligned}
$$

In turn, by considering (7) and (22), the variation $\widetilde{\delta}_{\perp} R_{i j}$ after a straightforward computation is

$$
\begin{aligned}
\widetilde{\delta}_{\perp} R_{i j} & =-2 J_{(i}^{a b} \widetilde{\nabla}_{\mid a} \widetilde{\nabla}_{b \mid} \Phi_{j)}+2 K_{(i} R_{j) l} \Phi^{l}-2 K_{i} K_{j} K_{l} \Phi^{l} \\
& +2 K^{a}{ }_{b i} K^{b}{ }_{c j} K^{c}{ }_{a l} \Phi^{l},
\end{aligned}
$$

or, collecting the last three terms in terms of $J_{i}^{a b}$ we are finally led to

$$
\widetilde{\delta}_{\perp} R_{i j}=-2 J_{(i}^{a b} \widetilde{\nabla}_{\mid a} \widetilde{\nabla}_{b \mid} \Phi_{j)}-2 K_{b(i}^{a} J_{j)}^{b c} K_{c a l} \Phi^{l} .
$$

On consistency grounds, let us focus attention in the case of $i=1$. In such a case $\widetilde{\delta}_{\perp} R_{i j} \rightarrow \delta_{\perp} \mathcal{R}=-2 \mathcal{R}_{a b} K^{a b} \Phi$, up to a boundary term, as expected [20], where $\mathcal{R}_{a b}=$ $K^{i} K_{a b i}-K_{a c}^{i} K_{b i}^{c}$ is the worldsheet Ricci tensor.

Regarding $\widetilde{\delta}_{\perp}\left(\widetilde{\Delta} \widehat{K}^{i}\right)$, we will consider the general expression to compute the deformation of the d'Alembertian operator applied to an arbitrary normal frame vector, $\Psi^{i}, 20$ ]

$$
\begin{aligned}
\widetilde{\delta}_{\perp}\left(\widetilde{\Delta} \widehat{K}^{i}\right) & =\widetilde{\Delta}\left(\widetilde{\delta}_{\perp} \widehat{K}^{i}\right)-2 \widetilde{\nabla}_{a}\left(K_{j}^{a b} \Phi^{j} \widetilde{\nabla}_{b} \widehat{K}^{i}\right) \\
& +\nabla^{a}\left(K_{j} \Phi^{j}\right) \widetilde{\nabla}_{a} \widehat{K}^{i}+2 K^{a b[i}\left(\widetilde{\nabla}_{a} \Phi^{j]}\right) \widetilde{\nabla}_{b} K_{j} \\
& +2 \widetilde{\nabla}_{a}\left[K^{a b[i}\left(\widetilde{\nabla}_{b} \Phi^{j]}\right) \widehat{K}_{j}\right] .
\end{aligned}
$$

Using the contracted Codazzi-Mainardi integrability condition we can put (25) as

$$
\begin{aligned}
\widetilde{\delta}_{\perp}\left(\widetilde{\Delta} \widehat{K}^{i}\right) & =\widetilde{\Delta}\left(\widetilde{\delta}_{\perp} \widehat{K}^{i}\right)-2 K_{j}^{a b} \widetilde{\nabla}_{a} \widehat{K}^{i} \widetilde{\nabla}_{b} \Phi^{j} \\
& +2 K^{a b i} \widetilde{\nabla}_{a} \widehat{K}_{j} \widetilde{\nabla}_{b} \Phi^{j}-2 K_{j}^{a b} \widetilde{\nabla}_{a} \widehat{K}^{j} \widetilde{\nabla}_{b} \Phi^{i} \\
& +\widetilde{\nabla}^{a} K^{i} \widehat{K}_{j} \widetilde{\nabla}_{a} \Phi^{j}-\widetilde{\nabla}^{a} K_{j} \widehat{K}^{j} \widetilde{\nabla}_{a} \Phi^{i} \\
& +2 K^{a b[i} \widetilde{\nabla}_{a} \widetilde{\nabla}_{b} \Phi^{j]} \widehat{K}_{j}-2 K_{j}^{a b} \widetilde{\nabla}_{a} \widetilde{\nabla}_{b} \widehat{K}^{i} \Phi^{j} \\
& +\widetilde{\nabla}^{a} \widehat{K}^{i} K_{j} \Phi^{j}-\widetilde{\nabla}_{a} \widehat{K}^{i} \widetilde{\nabla}^{a} K_{j} \Phi^{j} .
\end{aligned}
$$

By collecting the variations (19), (24) and (26) into (14) we obtain

$$
\begin{aligned}
\delta_{\perp}^{2} S & =\int_{m} d A\left[\widetilde{\delta}_{\perp}\left(\widetilde{\Delta} \widehat{K}_{i}\right)-\widetilde{\delta}_{\perp} R_{i j} \widehat{K}^{j}-R_{i j} \widetilde{\delta}_{\perp} \widehat{K}^{j}\right] \Phi^{i}, \\
& =\int_{m} d A \Phi^{i}\left[\widetilde{\Delta}\left(\widetilde{\delta}_{\perp} \widehat{K}_{i}\right)-2 K_{j}^{a b} \widetilde{\nabla}_{a} \widehat{K}_{i} \widetilde{\nabla}_{b} \Phi^{j}+2 K_{i}^{a b} \widetilde{\nabla}_{a} \widehat{K}_{j} \widetilde{\nabla}_{b} \Phi^{j}-2 K_{j}^{a b} \widetilde{\nabla}_{a} \widehat{K}^{j} \widetilde{\nabla}_{b} \Phi_{i}+\widetilde{\nabla}^{a} K_{i} \widehat{K}_{j} \widetilde{\nabla}_{a} \Phi^{j}\right. \\
& -\widetilde{\nabla}^{a} K_{j} \widehat{K}^{j} \widetilde{\nabla}_{a} \Phi_{i}+2 K_{[i}^{a b} \widetilde{\nabla}_{\mid a} \widetilde{\nabla}_{b \mid} \Phi_{j]} \widehat{K}^{j}-2 K_{j}^{a b} \widetilde{\nabla}_{a} \widetilde{\nabla}_{b} \widehat{K}_{i} \Phi^{j}+\widetilde{\nabla}^{a} \widehat{K}_{i} K_{j} \Phi^{j}-\widetilde{\nabla}_{a} \widehat{K}_{i} \widetilde{\nabla}^{a} K_{j} \Phi^{j} \\
& \left.+2 J_{(i}^{a b} \widetilde{\nabla}_{\mid a} \widetilde{\nabla}_{b \mid} \Phi_{j)} \widehat{K}^{j}+2 K_{b(i}^{a} J_{j)}^{b c} K_{c a l} \widehat{K}^{j} \Phi^{l}+\frac{1}{\sqrt{K^{r} K_{r}}} R_{i l} \Pi^{l j}\left(\widetilde{\Delta} \Phi_{j}-R_{j m} \Phi^{m}\right)\right] .
\end{aligned}
$$

From now on, the factor $(-\alpha)$ will be omitted in the writing of the work, for short. To continue, we analyze the first term in (27)

$$
\delta_{\perp}^{2} S_{1}:=\int_{m} d A \Phi^{i} \widetilde{\Delta}\left(\widetilde{\delta}_{\perp} \widehat{K}_{i}\right)=\int_{m} d A \widetilde{\Delta} \Phi^{i} \widetilde{\delta}_{\perp} \widehat{K}_{i},
$$

where we have neglected divergence terms. Inserting now the variation (19) into this expression and continuing integrating by parts as well as ignoring the divergence 
terms, we get

$$
\begin{aligned}
\delta_{\perp}^{2} S_{1} & =\int_{m} d A \Phi^{i}\left[-\widetilde{\Delta}\left(\frac{1}{\sqrt{K^{r} K_{r}}} \Pi_{i j} \widetilde{\Delta} \Phi^{j}\right)\right. \\
& \left.+\frac{1}{\sqrt{K^{r} K_{r}}} R_{i l} \Pi^{l j} \widetilde{\Delta} \Phi_{j}\right]
\end{aligned}
$$

Now, by substituting (28) into (27) we can write the second variation of the action as

$$
\begin{aligned}
\delta_{\perp}^{2} S & =\int_{m} d A \Phi^{i}\left[-\widetilde{\Delta}\left(\frac{1}{\sqrt{K^{r} K_{r}}} \Pi_{i j} \widetilde{\Delta}^{j} \Phi^{j}\right)\right. \\
& +\frac{2}{\sqrt{K^{r} K_{r}}} R_{i l} \Pi^{l j} \widetilde{\Delta} \Phi_{j}-2 K_{j}^{a b} \widetilde{\nabla}_{a} \widehat{K}_{i} \widetilde{\nabla}_{b} \Phi^{j} \\
& +2 K_{i}^{a b} \widetilde{\nabla}_{a} \widehat{K}_{j} \widetilde{\nabla}_{b} \Phi^{j}-2 K_{j}^{a b} \widetilde{\nabla}_{a} \widehat{K}^{j} \widetilde{\nabla}_{b} \Phi_{i} \\
& +\widetilde{\nabla}^{a} K_{i} \widehat{K}_{j} \widetilde{\nabla}_{a} \Phi^{j}-\widetilde{\nabla}^{a} K_{j} \widehat{K}^{j} \widetilde{\nabla}_{a} \Phi_{i} \\
& +2 K_{[i}^{a b} \widetilde{\nabla}_{\mid a} \widetilde{\nabla}_{b \mid} \Phi_{j]} \widehat{K}^{j}-2 K_{j}^{a b} \widetilde{\nabla}_{a} \widetilde{\nabla}_{b} \widehat{K}_{i} \Phi^{j} \\
& -\widetilde{\nabla}_{a} \widehat{K}_{i} \widetilde{\nabla}^{a} K_{j} \Phi^{j}+\widetilde{\nabla}^{a} \widehat{K}_{i} K_{j} \Phi^{j} \\
& +2 J_{(i}^{a b} \widetilde{\nabla}_{\mid a} \widetilde{\nabla}_{b \mid} \Phi_{j)} \widehat{K}^{j}+2 K^{a}{ }_{b(i} J_{j)}^{b c} K_{c a l} \widehat{K}^{j} \Phi^{l} \\
& \left.-\frac{1}{\sqrt{K^{r} K_{r}}} R_{i l} \Pi^{l j} R_{j m} \Phi^{m}\right] .
\end{aligned}
$$

In order to reduce this to a more familiar form, we will work some of the terms in the third and fourth lines of this expression

$$
\begin{aligned}
-2 K_{j}^{a b} \widetilde{\nabla}_{a} \widetilde{\nabla}_{b} \widehat{K}_{i} & -\widetilde{\nabla}_{a} \widehat{K}_{i} \widetilde{\nabla}^{a} K_{j}=2 J_{j}^{a b} \widetilde{\nabla}_{a} \widetilde{\nabla}_{b} \widehat{K}_{i} \\
& -\widetilde{\nabla}^{a}\left(K_{j} \widetilde{\nabla}_{a} \widehat{K}_{i}\right)-K_{j} R_{i l} \widehat{K}^{l},
\end{aligned}
$$

and

$$
\begin{aligned}
2\left(J_{(i}^{a b} \widetilde{\nabla}_{\mid a} \widetilde{\nabla}_{b \mid} \Phi_{j)}\right. & \left.+2 K_{[i}^{a b} \widetilde{\nabla}_{\mid a} \widetilde{\nabla}_{b \mid} \Phi_{j]}\right) \widehat{K}^{j} \\
& =2 \delta_{i j} J_{l}^{a b} \widehat{K}^{l} \widetilde{\nabla}_{a} \widetilde{\nabla}_{b} \Phi^{j}-\sqrt{K^{r} K_{r}} \Pi_{i j} \widetilde{\Delta} \Phi^{j}
\end{aligned}
$$

where we have considered the equations of motion (12).
Substituting these into the variation (29) yields

$$
\begin{aligned}
\delta_{\perp}^{2} S & =\int_{m} d A \Phi^{i}\left[-\widetilde{\Delta}\left(\frac{1}{\sqrt{K^{r} K_{r}}} \Pi_{i j} \widetilde{\Delta} \Phi^{j}\right)\right. \\
& +\frac{2}{\sqrt{K^{r} K_{r}}} R_{i l} \Pi^{l j} \widetilde{\Delta} \Phi_{j}-\sqrt{K^{r} K_{r}} \Pi_{i j} \widetilde{\Delta} \Phi^{j} \\
& +2 \delta_{i j} J_{l}^{a b} \widetilde{\nabla}_{a} \widehat{K}^{l} \widetilde{\nabla}_{b} \Phi^{j}+2 \delta_{i j} J_{l}^{a b} \widehat{K}^{l} \widetilde{\nabla}_{a} \widetilde{\nabla}_{b} \Phi^{j} \\
& -\delta_{i j} \widehat{K}_{l} \widetilde{\nabla}^{a} K^{l} \widetilde{\nabla}_{a} \Phi^{j}+4 K_{[i}^{a b} \widetilde{\nabla}_{|a|} \widehat{K}_{j]} \widetilde{\nabla}_{b} \Phi^{j} \\
& +\widetilde{\nabla}^{a} K_{i} \widehat{K}_{j} \widetilde{\nabla}_{a} \Phi^{j}+\widetilde{\nabla}^{a} \widehat{K}_{i} K_{j} \widetilde{\nabla}_{a} \Phi^{j} \\
& \left.+\left(2 J_{i}^{a b} \widetilde{\nabla}_{a} \widetilde{\nabla}_{b} \widehat{K}_{j}-\widetilde{\nabla}^{a} K_{j} \widetilde{\nabla}_{a} \widehat{K}_{i}-G_{i j}\right) \Phi^{j}\right]
\end{aligned}
$$

where, once again, we have used the equations of motion (12) and introduced the following tensor in the normal frame

$$
G_{i j}:=\frac{1}{\sqrt{K^{r} K_{r}}} R_{i l} \Pi^{l m} R_{m j}-2 K_{b(i}^{a} J_{l)}^{b c} K_{c a j} \widehat{K}^{l} .
$$

The remarkable thing about the tensor $G_{i j}$ is that it does not contain derivatives neither of the unitary vector $\widehat{K}^{i}$ nor the breathing deformation field $\Phi^{i}$. Now, an integration by parts in the second line of Eq. (30) yields

$$
\begin{aligned}
\int_{m} d A \Phi^{i} 2 \delta_{i j} J_{l}^{a b} \widetilde{\nabla}_{a} \widehat{K}^{l} \widetilde{\nabla}_{b} \Phi^{j} & = \\
& -\int_{m} d A \Phi^{i} \delta_{i j} J_{l}^{a b} \widetilde{\nabla}_{a} \widetilde{\nabla}_{b} \widehat{K}^{l} \Phi^{j}
\end{aligned}
$$

up to a boundary term. The expression (30) is finally rearranged in the covariant form

$$
\delta_{\perp}^{2} S=\int_{m} d A \Phi^{i} \mathcal{L}_{i j} \Phi^{j},
$$

where the local differential operators $\mathcal{L}_{i j}$ are given by

$$
\begin{aligned}
\mathcal{L}_{i j} \Phi^{j} & =-\widetilde{\Delta}\left(\frac{1}{\sqrt{K^{r} K_{r}}} \Pi_{i j} \widetilde{\Delta} \Phi^{j}\right)+\frac{2}{\sqrt{K^{r} K_{r}}} R_{i l} \Pi^{l j} \widetilde{\Delta}_{j}-\sqrt{K^{r} K_{r}} \Pi_{i j} \widetilde{\Delta}^{j}+2 \delta_{i j} J_{l}^{a b} \widehat{K}^{l} \widetilde{\nabla}_{a} \widetilde{\nabla}_{b} \Phi^{j} \\
& -\delta_{i j} \widehat{K}_{l} \widetilde{\nabla}^{a} K^{l} \widetilde{\nabla}_{a} \Phi^{j}+4 K_{[i}^{a b} \widetilde{\nabla}_{|a|} \widehat{K}_{j]} \widetilde{\nabla}_{b} \Phi^{j}+\widetilde{\nabla}^{a} K_{i} \widehat{K}_{j} \widetilde{\nabla}_{a} \Phi^{j}+\widetilde{\nabla}^{a} \widehat{K}_{i} K_{j} \widetilde{\nabla}_{a} \Phi^{j} \\
& +\left(2 J_{i}^{a b} \widetilde{\nabla}_{a} \widetilde{\nabla}_{b} \widehat{K}_{j}-\delta_{i j} J_{l}^{a b} \widetilde{\nabla}_{a} \widetilde{\nabla}_{b} \widehat{K}^{l}-\widetilde{\nabla}^{a} K_{j} \widetilde{\nabla}_{a} \widehat{K}_{i}-G_{i j}\right) \Phi^{j}
\end{aligned}
$$

From another point of view, the second-order variation (32) can be seen as a related deformation $X^{\mu} \rightarrow$ $X^{\mu}\left(x^{a}\right)+\Phi^{\prime i} n^{\mu}{ }_{i}$ applied to the first variation of the action, and subsequently imposing $\Phi^{\prime i}=\Phi^{i}$. The system (33) consist of $N-2$ highly non-trivial, coupled partial differential equations for the $\Phi^{i}$. Unfortunately, we face with fourth-order differential equations for $\Phi^{i}$ where the analytical solutions are hard to obtain. These expressions comprise what are known as Jacobi equations for the case of the gonihedric string field theory.

Some aspects of these Jacobi equations are in order. Geometrically, this set of relations describes the behaviour of this type of surfaces which are close or in the neighborhood of a reference surface one. These equations are explicitly covariant under local normal frame rotations. On the other hand, on physical grounds, the solutions for this set of equations address the question of stability through the nature of the breathing modes, $\Phi^{i}$, of the worldsheet. There is the hunch that some of 
these Jacobi equations are pure gauge or not contribute to the stability analysis since we originally have a secondorder derivative theory which gives rise to some spurious geometric degrees of freedom. This fact will supported by a constraint Hamiltonian analysis for the model [29]. Anyhow, this idea deserves further attention. We would like to go one step further in the understanding of the stability for surfaces when this type of nontrivial rigidity is present, but at this stage we do not have strong geometric arguments to provide an answer due to the high degree of complexity of the equations (33). In fact, we expect that an unstable behaviour predominates due to the close relationship of this model with a Nambu-Goto model, in an extended space [31], which is known to have the characteristic of being unstable. This issue also deserves special care.

One immediate approximation is given by $\widetilde{\nabla}_{a} K^{i}=0$. In this case we have $R_{i j} \widehat{K}^{j}=J_{i}^{a b} K_{a b j} \widehat{K}^{j}=0$. It is believed that this limit may have impact on the short wavelength fluctuations around classical trajectories given by $K_{j} K^{j} K^{i}=K_{a b}^{i} K_{j}^{a b} K^{j}$. For this case the Jacobi equations (33) read

$$
\begin{aligned}
\mathcal{L}_{i j} \Phi^{j} & =-\frac{1}{\sqrt{K^{r} K_{r}}} \Pi_{i j} \widetilde{\Delta} \widetilde{\Delta} \Phi^{j}+\frac{2}{\sqrt{K^{r} K_{r}}} R_{i l} \Pi^{l}{ }_{j} \widetilde{\Delta} \Phi^{j} \\
& -\sqrt{K^{r} K_{r}} \Pi_{i j} \widetilde{\Delta} \Phi^{j}+2 \delta_{i j} J_{l}^{a b} \widehat{K}^{l} \widetilde{\nabla}_{a} \widetilde{\nabla}_{b} \Phi^{j} \\
& -G_{i j} \Phi^{j} .
\end{aligned}
$$

As before, consistency in the full set of results is mandatory. To show this note first that the tensor (31) can be put in the fashion

$$
\begin{aligned}
G_{i j} & =\frac{1}{\sqrt{K^{r} K_{r}}} R_{i l} \Pi^{l m} R_{m j}+\sqrt{K^{r} K_{r}} R_{i j}-K_{i} R_{j l} \widehat{K}^{l} \\
& -2 J_{i}^{a b} K_{b c j} K_{a l}^{c} \widehat{K}^{l} .
\end{aligned}
$$

Then, for a hypersurface embbeding we get $G_{11}=$ $-2 J^{a b} K_{a}{ }^{c} K_{b c}$, where $J^{a b}=g^{a b} K-K^{a b}$. For this particular case, from (33) we have

$$
\mathcal{L} \Phi=2\left(J^{a b} \nabla_{a} \nabla_{b} \Phi-J^{a b} K_{a}{ }^{c} K_{b c} \Phi\right)=0 .
$$

We have thus encountered a second-order differential equation for $\Phi$. This equation is clearly in accord with the results found in Ref. 23] for the case of an action functional depending linearly of the trace of the extrinsic cur- vature which corresponds to the so-called second Lovelock type brane invariant. In another fashion, Eq. (36) reads

$$
J^{a b} \nabla_{a} \nabla_{b} \Phi-M^{2} \Phi=0,
$$

which looks like a type wave equation with a mass-like term of the form $M^{2}:=J^{a b} K_{a}{ }^{c} K_{b c}=\mathcal{R}_{a b} K^{a b}$, with $\mathcal{R}_{a b}$ being the worldsheet Ricci tensor.

\section{CONCLUDING REMARKS}

In this paper we have presented a covariant approach for the analysis of perturbations on surfaces governed by the so-called gonihedric string field theory. We have mainly focused on the normal deformations since these represent the only physically perturbations. According to the Savvidy sorting, we opt to develop the Model A for this theory with the idea of maintain the original field variables and of exploiting the natural geometric structures associated to the worldsheet. Though the square root Lagrangian seems complicated, we can handle it and exhibit in an elegant fashion by means of the unit vector $\widehat{K}^{i}$. Regarding this point, owing to inner geometrical nature of the action (1), the introduction of the unit vector exhibits the extreme elegance and simplicity of the eom (9) and helps to simplify, in some sense, the form of the stability conditions for the model. The generalization of the action (11) to describe $p$-dimensional extended objects is straightforward and the geometric analysis can be carried out following similar lines obtaining analogous conclusions. In this regard, there is a related approach to discuss the concept of rigidity in quantum gravity in relation with the so-called gonihedric principle by describing the propagation of compact orientable random branes with no spatial boundary 32,35$]$.

\section{ACKNOWLEDGMENTS}

Enlightening remarks and discussions with Miguel Cruz are acknowledged. This work was partially supported by Sistema Nacional de Investigadores, México. Also, partial support from ProDeP-2017-México is acknowledged.
[1] R. V. Ambartzumanian, G. K. Savvidy, K. G. Savvidy and G. S. Sukiasian, Phys. Lett. B 275 (1992) 99-102.

[2] G. K. Savvidy and K. G. Savvidy, Mod. Phys. Lett. A 8 (1992) 2963-2971.

[3] G. K. Savvidy and K. G. Savvidy, Int. J. Mod. Phys. A 8 (1993) 3993-4011.

[4] G. K. Savvidy and R. Manvelyan, Phys. Lett. B 533 (2002) 138-145.
[5] A. R. Fazio and G. K. Savvidy, Mod. Phys. Lett. A 18 (2003) 2817-2828.

[6] G. K. Savvidy, Int. J. Mod. Phys. A 19 (2004) 3171-3194.

[7] G. K. Savvidy and F. J. Wegner, Nucl. Phys. B 413 (1994) 605-613.

[8] G. K. Savvidy, K. G. Savvidy and F. J. Wegner, Nucl. Phys. B 443 (1995) 565-580.

[9] G. Savvidy, Mod. Phys. Lett. B 29 (2015) 1550203. 
[10] G. Savvidy, Gravity with linear action and gravitational singularities, arXiv:1705.01459 [hep-th].

[11] A. R. Fazio, Acta Phys. Pol. B 34 4825-4834 (2003).

[12] G. Arreaga, R. Capovilla and J. Guven, Annals of Phys. 279 (2000) 126-158.

[13] B. Carter, Brane dynamics for treatment of cosmic strings and vortons, in "Recent Developments in Gravitation and Mathematics", Proc. 2nd Mexican School on Gravitation and Mathematical Physics, eds. A. Garcia, C. Lammerzahl, A. Macias, T. Matos and D. Nuñez. (Science Network Publishing, Konstanz, 1997); hep-th/9705172

[14] J. Mourad, "Continous spin and tensionless strings", arXiv:hep-th/0410009.

[15] J. Mourad, AIP Conf. Proc. 861 (2006) 436-443.

[16] B. Y. Cheng, Geometry of Submanifolds (Dekker, 1973).

[17] M. Dajczer, Submanifolds and Isometric Immersions (Publish or Perish, 1990).

[18] J. Guven, Phys. Rev. D 48, 4606 (1993).

[19] G. K. Savvidy, Phys. Lett. B 552 (2003) 72-80.

[20] R. Capovilla and J. Guven, Phys. Rev. D 51 (1995) 67366743.

[21] S. Kobayashi and K. Nomizu, Foundations of Differential Geometry: Volume II (Interscience, New York, 1969).

[22] M. Cruz and E. Rojas, Class. Quant. Grav. 30 (2013) 115012.

[23] N. Bagatella-Flores, C. Campuzano, M. Cruz and E. Rojas, Class. Quant. Grav. 33 (2016) 245012.
[24] S. Svetina and B. Žekš, Eur. Biophys. J. 17 (1989) 101111.

[25] R. Capovilla, J. Guven and J. A. Santiago, J. Phys. A: Math. Gen. 38 (2003) 6281-6295.

[26] R. Capovilla and J. Guven, J. Phys. A: Math. Gen. 37 (2004) 5983-6001.

[27] R. Capovilla, J. Guven and E. Rojas, J. Phys. A: Math. Gen. 38 (2005) 8201-8210.

[28] R. Capovilla, J. Guven and E. Rojas, J. Phys. A: Math. Gen. 38 (2005) 8841-8860.

[29] E. Rojas, Ostrogradsky Hamiltonian analysis of the gonihedric string theory. In preparation.

[30] H. A. Schwarz, Gesammelte Mathematische Abhandlungen. Erster Band. (Springer-Verlag Berlin Heidelberg, 1890).

[31] G. Savvidy, Phys. Lett. B 615 (2005) 285-290.

[32] G. K. Savvidy and K. G. Savvidy, Mod. Phys. Lett. A 11 (1996) 1379-1396.

[33] J. Ambjorn, G. K. Savvidy and K. G. Savvidy, Nucl. Phys. B 486 (1997) 390-412.

[34] G. K. Savvidy, Nucl. Phys. B Proc. Suppl. 57 (1997) 104114.

[35] C. Campuzano, R. Capovilla, A. Cervantes and E. Rojas, AIP Conf.Proc. 1420 (2012) 42-46. 